\title{
Model systems to define the role of AKR1D1 in metabolic liver disease
}

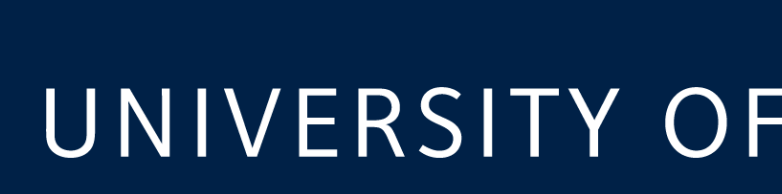 OXFORD}

Nikolaos Nikolaou1, Laura Gathercole ${ }^{1}$, James Dunford², Wenhwa Lee ${ }^{2}$, Reina Lim³ Jane McKeating ${ }^{3}$, Udo Oppermann ${ }^{2}$, Leanne Hodson ${ }^{1}$ and Jeremy Tomlinson ${ }^{1}$

1 University of Oxford, Oxford Centre for Diabetes, Endocrinology and Metabolism, United Kingdom

2University of Oxford, Nuffield Department of Orthopaedics, Rheumatology and Musculoskeletal Sciences, United Kingdom

${ }^{3}$ University of Birmingham, School of Immunity and Infection, United Kingdom

\section{Background}

Non-alcoholic fatty liver disease is the hepatic manifestation of the global epidemic of metabolic disease. It is tightly associated with obesity and type 2 diabetes, yet the precise mechanisms that drive its aetiology are not fully defined. Steroid hormones, including glucocorticoids and sex steroids, regulate metabolic phenotype; additionally, bile acids have recently been identified as potent metabolic regulators. AKR1D1 (5ß-reductase), is predominantly expressed in the liver, and is a crucial regulator of steroid hormone clearance as well as bile acid synthesis. Its role in pathogenesis of metabolic disease has not been examined. We therefore developed systems to define the enzymology of human AKR1D1 in cell free assays and to determine the impact of manipulation of AKR1D1 expression and activity in human hepatocyte models.

\section{Methods}

B21 Rosetta bacteria cells were transformed with an AKR1D1 construct (pNIC-CTHF+AKR1D1) and recombinant protein extracted and purified. A high throughput assay was developed to determine AKR1D1 activity, substrate specificity and enzyme kinetics. 9 different substrates (7 steroids and 2 bile acid intermediates) were tested against the purified enzyme and activity was measured by NADPH reduction in a dose and time dependent manner. AKR1D1 mRNA expression was characterized in 4 different hepatoma cell lines (Hep3b, HepG2, C3A and Huh7.0) as well as primary cultures of human hepatocytes. In addition, HepG2 cells were differentiated using an established protocol (including 1\% DMSO treatment), and gene expression analyzed after 7, 14 and 21 days. Overexpression and siRNA knock down of AKR1D1 in HepG2 cells were also performed. AKR1D1 was highly expressed in HepG2 cells and expression decreased across differentiation, to levels that were similar to those seen in primary cultures of human hepatocytes. Successful over-expression and knock down of AKR1D1 were confirmed in HepG2 cells using real-time PCR. Importantly, changes in gene expression were paralleled by functional activity as measured by progesterone clearance.

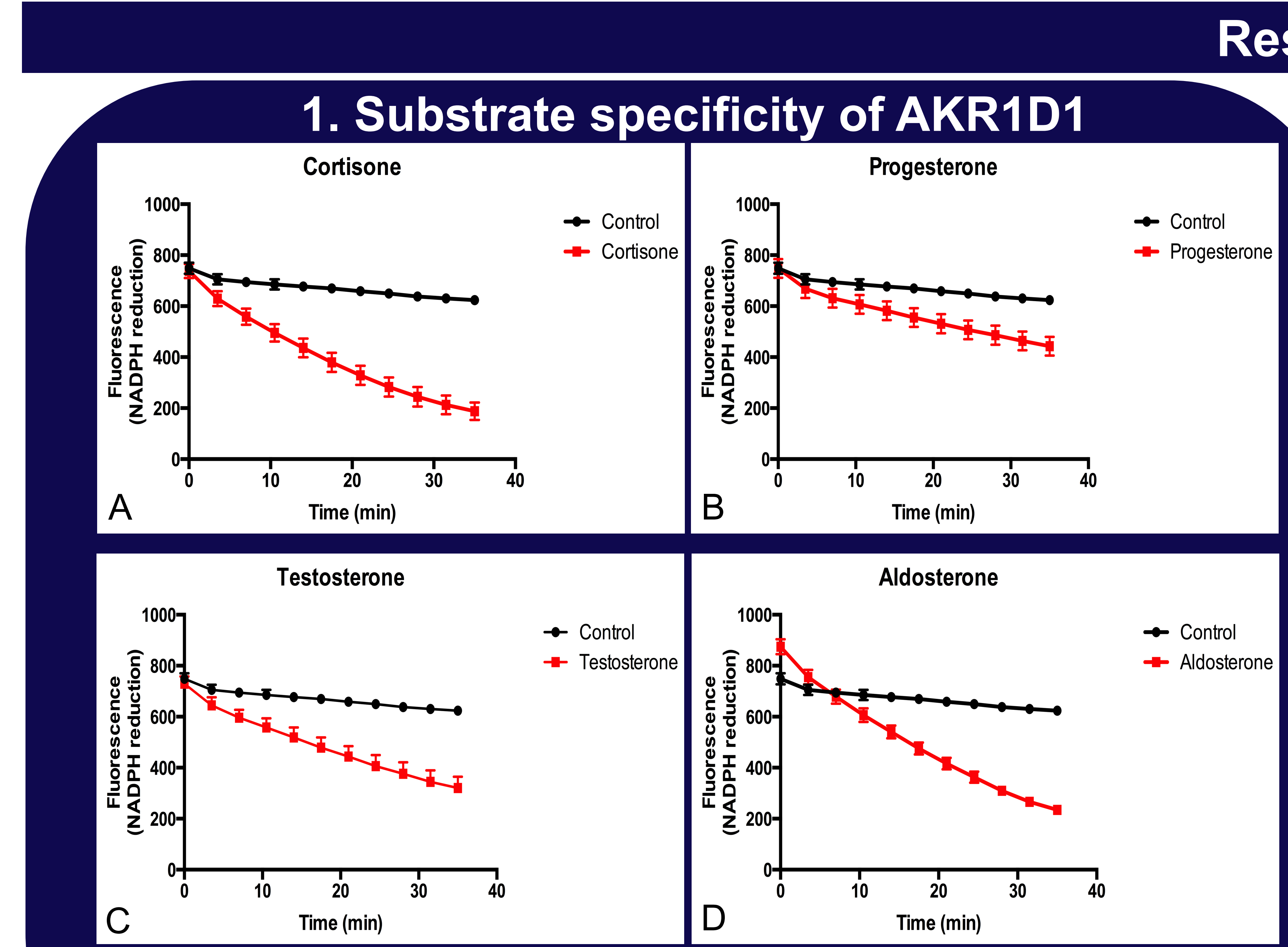

Figure 1: AKR1D1 activity measured by NADPH reduction (FU) using Cortisone (A), Progesterone (B), Testosterone (C) and Aldosterone (D) as a substrate.

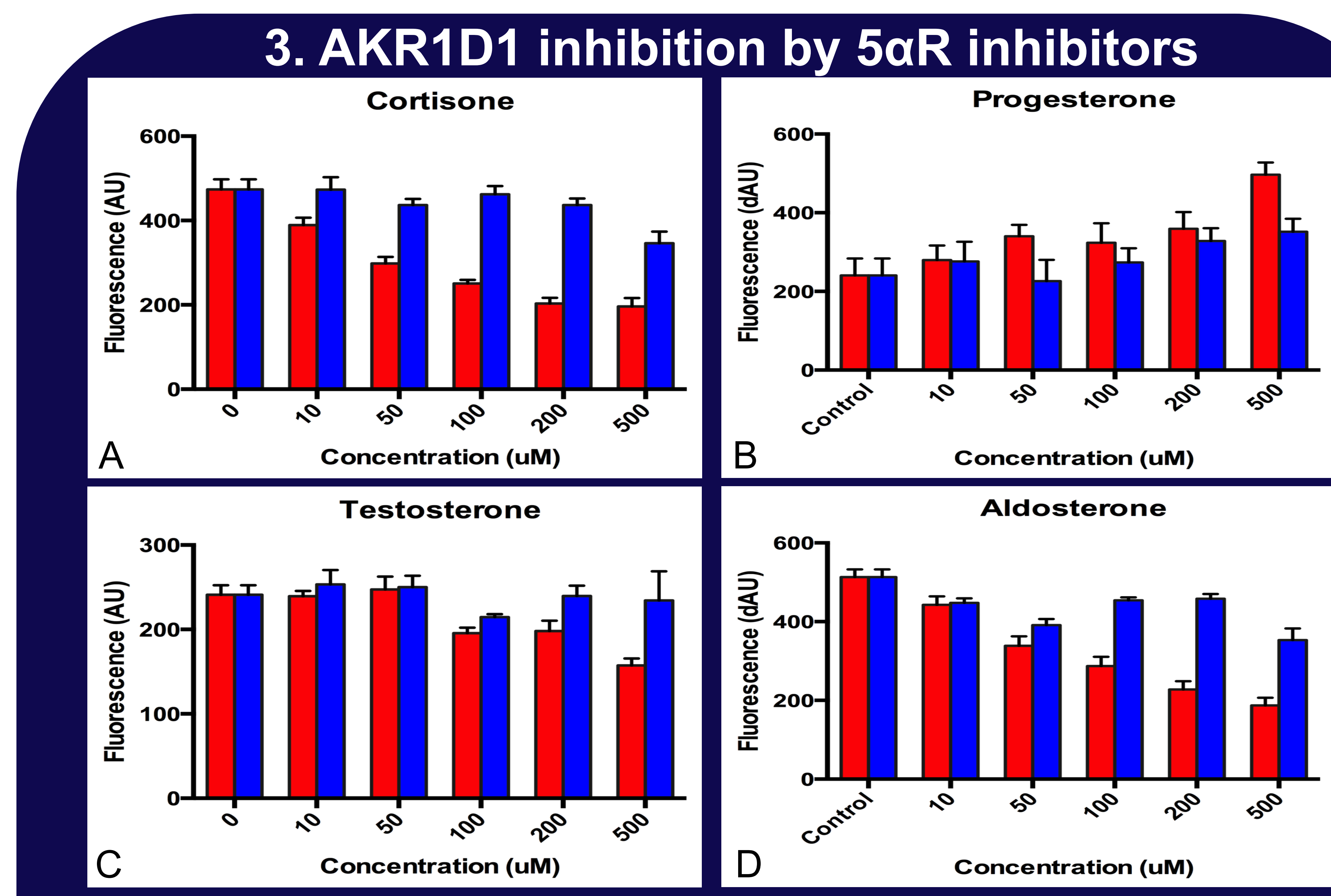

Figure 3: Inhibition of AKR1D1 activity by Finasteride (red bar) and Dutasteride (blue bar) using Cortisone (A), Progesterone (B), Testosterone (C) and Aldosterone (D) as a substrate, measured by NADPH reduction (FU).
Results

\begin{tabular}{|c|c|c|c|}
\hline \multicolumn{4}{|c|}{ 2. Enzyme kinetics of AKR1D1 } \\
\hline Substrate & Km & Kcat & Kcat/Km \\
\hline Androstenedione & 3.1 & 0.35 & 0.113 \\
\hline Progesterone & 3.29 & 0.25 & 0.077 \\
\hline Corticosterone & 4.36 & 0.30 & 0.069 \\
\hline Cortisone & 5.44 & 0.73 & 0.134 \\
\hline Aldosterone & 6.65 & 0.83 & 0.124 \\
\hline Testosterone & 7.2 & 0.73 & 0.101 \\
\hline 7a,12a dihydroxy & 15.82 & 0.15 & 0.009 \\
\hline cholestenone & 25.42 & 0.46 & 0.018 \\
\hline Cortisol & 25.77 & 0.24 & 0.009 \\
\hline 7a hydroxy cholestenone & & \\
\hline
\end{tabular}

Figure 2: $\mathrm{Km}$, Kcat and $\mathrm{Kcat} / \mathrm{Km}$ values for all AKR1D1 substrates as measured by high throughput screening assay.

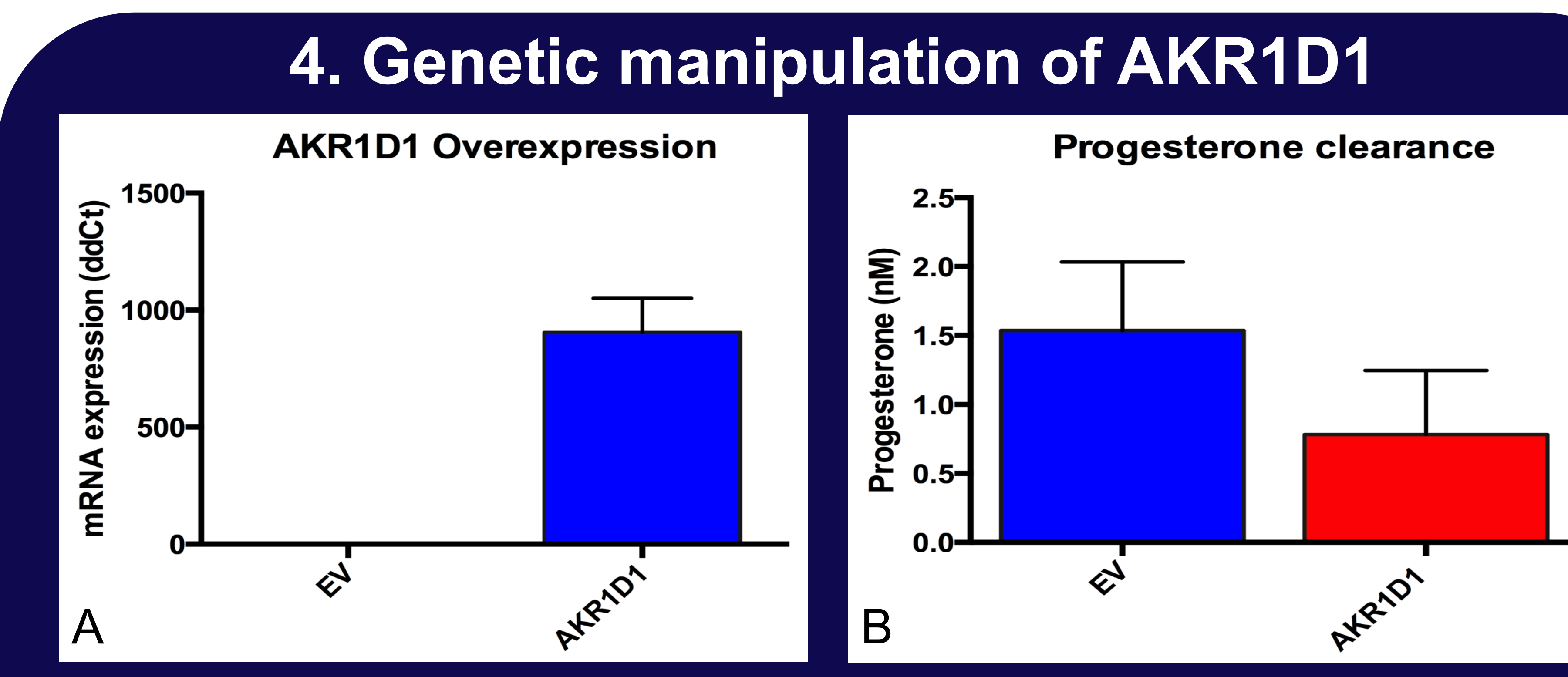

Figure 4: AKR1D1 overexpression in HepG2 cells, as measured by real-time PCR $(A)$ and progesterone clearance $(B)$.

5. HepG2 differentiation by DMSO treatment
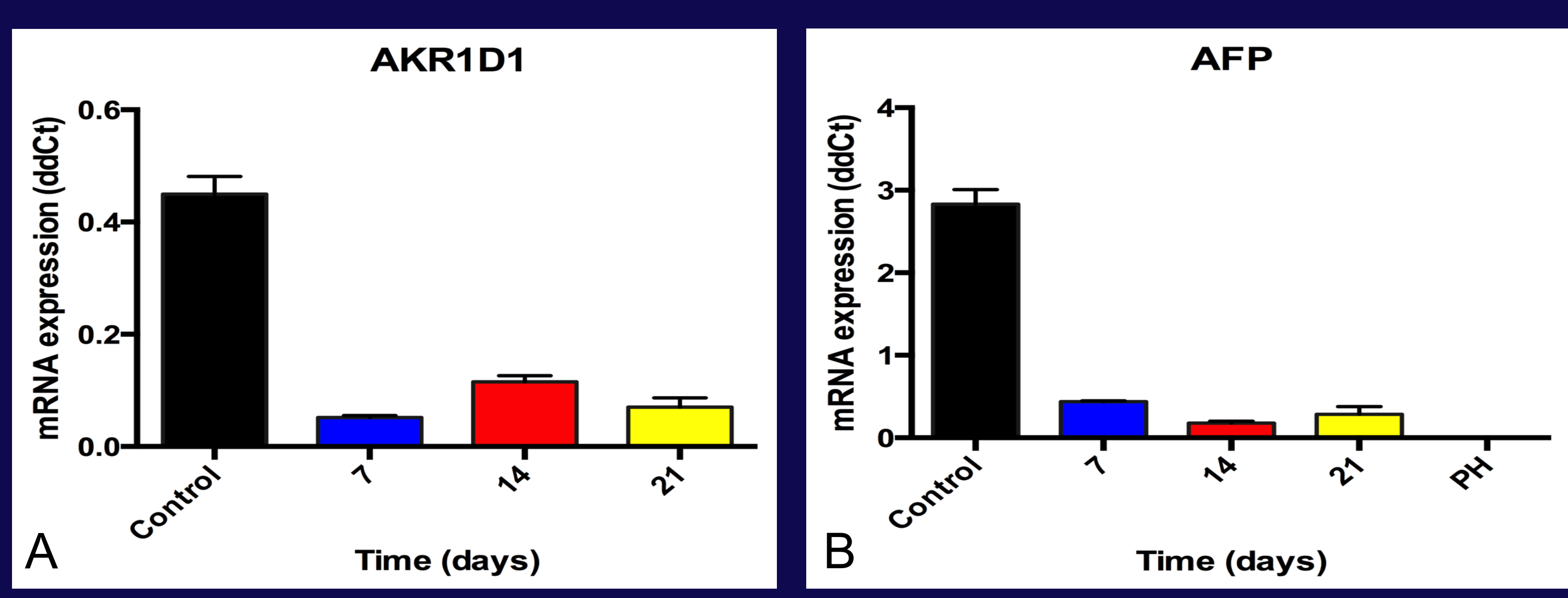

Figure 5: AKR1D1 (A) and AFP (B) mRNA expression after differentiation with $1 \%$ DMSO as measured by real-time $P C R$. 